\title{
In silico Hierarchical Clustering of Neuronal Populations in the Rat Ventral Tegmental Area Based on Extracellular Electrophysiological Properties
}

\author{
Mathieu Di Miceli ${ }^{1,2 * \dagger}$, Zoé Husson 2,3,4†, Philippe Ruel ${ }^{5}$, Sophie Layé2, Daniela Cota ${ }^{3}$, \\ Xavier Fioramonti ${ }^{2}$, Clémentine Bosch-Bouju ${ }^{2}$ and Benjamin Gronier ${ }^{1}$ \\ ${ }^{1}$ Pharmacology and Neuroscience Research Group, Leicester School of Pharmacy, De Montfort University, Leicester, United \\ Kingdom, ${ }^{2}$ Laboratoire NutriNeuro, UMR INRAE 1286, Université de Bordeaux, Bordeaux, France, ${ }^{3}$ INSERM, Neurocentre \\ Magendie, Physiopathologie de la Plasticité Neuronale, University of Bordeaux, Bordeaux, France, ${ }^{4}$ GG, Université de \\ Montpellier, CNRS, INSERM, Montpellier, France, ${ }^{5}$ Département de Mathématiques, Lycée Joffre, Académie de Montpellier, \\ Montpellier, France
}

OPEN ACCESS

Edited by:

Fu-Ming Zhou,

University of Tennessee Health Science Center (UTHSC),

United States

Reviewed by:

Mark S. Brodie,

University of lllinois at Chicago,

United States

Mostafa El Mansari,

Univeristy of Ottawa, Canada

*Correspondence:

Mathieu Di Miceli

mathieu.di-miceli@inrae.fr

${ }^{\dagger}$ These authors have contributed equally to this work

Received: 28 January 2020

Accepted: 15 July 2020

Published: 13 August 2020

Citation:

Di Miceli M, Husson Z, Ruel P, Layé S, Cota $D$, Fioramonti $X$,

Bosch-Bouju C and Gronier B

(2020) In silico Hierarchical Clustering of Neuronal Populations in the Rat Ventral Tegmental Area Based on Extracellular Electrophysiological Properties.

Front. Neural Circuits 14:51. doi: 10.3389/fncir.2020.00051
The ventral tegmental area (VTA) is a heterogeneous brain region, containing different neuronal populations. During in vivo recordings, electrophysiological characteristics are classically used to distinguish the different populations. However, the VTA is also considered as a region harboring neurons with heterogeneous properties. In the present study, we aimed to classify VTA neurons using in silico approaches, in an attempt to determine if homogeneous populations could be extracted. Thus, we recorded 291 VTA neurons during in vivo extracellular recordings in anesthetized rats. Initially, 22 neurons with high firing rates $(>10 \mathrm{~Hz})$ and short-lasting action potentials (AP) were considered as a separate subpopulation, in light of previous studies. To segregate the remaining 269 neurons, presumably dopaminergic (DA), we performed in silico analyses, using a combination of different electrophysiological parameters. These parameters included: (1) firing rate; (2) firing rate coefficient of variation (CV); (3) percentage of spikes in a burst; (4) AP duration; (5) $\Delta t_{1}$ duration (i.e., time from initiation of depolarization until end of repolarization); and (6) presence of a notched AP waveform. Unsupervised hierarchical clustering revealed two neuronal populations that differed in their bursting activities. The largest population presented low bursting activities ( $<17.5 \%$ of total spikes in burst), while the remaining neurons presented higher bursting activities (>17.5\%). Within nonhigh-firing neurons, a large heterogeneity was noted concerning AP characteristics. In conclusion, this analysis based on conventional electrophysiological criteria clustered two subpopulations of putative DA VTA neurons that are distinguishable by their firing patterns (firing rates and bursting activities) but not their AP properties.

Keywords: electrophysiology, VTA, hierarchical clustering, dopaminergic neurons, neurophysiology

Abbreviations: AP, action potential; PCA, principal component analysis; VTA, ventral tegmental area; CV, coefficient of variation; DA, dopaminergic. 


\section{INTRODUCTION}

Mesolimbic dopaminergic (DA) structures control motivation and reward (Taylor et al., 2016). Amongst midbrain DA structures, the ventral tegmental area (VTA) has been extensively studied. Outputs from the VTA include the ventral striatum and the anterior cortex (Beier et al., 2015). Recently, several studies have highlighted some heterogeneity amongst VTA neurons, especially concerning their input/output circuitry (Morales and Margolis, 2017; Poulin et al., 2018), their responses to DA pharmacology (Margolis et al., 2006, 2008), their electrophysiological characteristics (Luo et al., 2008) as well as their immunohistochemical signatures (Morales and Root, 2014; Yamaguchi et al., 2015). Therefore, the functions of the different neuronal subpopulations within VTA neurons are of interest.

DA neurons are extensively studied and, as such, electrophysiological identification of these neurons has been relying on several electrophysiological criteria. These include both firing patterns (such as firing rate, firing regularity and bursting activities) and action potential (AP) properties (width, shape, and presence of notched waveform), all summarized in a 2012 review by Ungless and Grace (2012). However, two studies identified putative DA neurons with electrophysiologically heterogeneous properties (Luo et al., 2008; Li et al., 2012).

In the present study, we investigated the heterogeneity of VTA neurons by combining all electrophysiological criteria in an attempt to classify neurons into different subpopulations. Here, we propose a simple and fast method for sorting non-high-firing neurons, presumably DA, that can be used during in vivo extracellular recordings, without requiring pharmacology or extensive analyses. First, high-firing neurons $(>10 \mathrm{~Hz})$ were considered as GABAergic neurons, following previous observations (Steffensen et al., 1998; Luo et al., 2008; Li et al., 2012). Since glutamatergic neurons account for 2-3\% of all neurons within the VTA (Nair-Roberts et al., 2008), we considered the remaining non-high-firing neurons as DA neurons. Hierarchical clustering in these neurons revealed 2 subpopulations of DA neurons. Remarkably, these subpopulations differed by their firing patterns, but not their AP characteristics.

\section{MATERIALS AND METHODS}

\section{Ethical Approval}

Experiments were performed in strict accordance with the recommendations in the Guide for the Care and Use of Laboratory Animals of the UK Home Office. All protocols were carried out under project and personal licenses issued by the UK Home Office under the UK Animals (Scientific Procedures) Act 1986 and were also approved by the Committee on the Ethics of Animal Experiments of De Montfort University (Protocol 60/4333). All experiments were performed under chloral hydrate anesthesia, administered at doses causing deep anesthesia (400 $\mathrm{mg} / \mathrm{kg}$ as a starting dose) as previously described (Grace and Bunney, 1983, 1984; Ackerman et al., 1993; Marinelli and White, 2000; Zhang et al., 2008; Valenti et al., 2012; Marinelli and McCutcheon, 2014). Anesthesia was maintained by administering small intravenous boluses $(\sim 0.03 \mathrm{~g} / \mathrm{kg})$, typically every 8-10 min. Depth of anesthesia was confirmed by the absence of withdrawal following paw and tail pinches.

\section{Animals}

Ninety-two adult male Sprague-Dawley rats $(P>56)$ were purchased from Charles River, UK. All animals were housed in groups of 2-4 per cage, maintained at $20-22^{\circ} \mathrm{C}$ with humidity rates above $40 \%$ under a $12: 12 \mathrm{~L} / \mathrm{D}$ cycle with lights $\mathrm{ON}$ at 07:00 h. Food and water were provided ad libitum. A cardboard tube was also placed in each cage to allow sheltering. Animals were allowed a 3-day acclimatization period after delivery. The 92 naive adult rats (250-450 g) included in the present study were also used in other studies with different objectives.

\section{In vivo Extracellular Single-Unit Electrophysiology}

Animals were deeply anesthetized and the procedure started when the absence of withdrawal responses to paw and tail pinches was achieved. Animals were secured to a stereotaxic frame and maintained at $36-37^{\circ} \mathrm{C}$. A catheter was inserted into the lateral tail vein to perform additional anesthetic administrations, if necessary, or to administer pharmacological agents. An incision was made across the top of the head and the edges of the skin drawn back to reveal the cranium. Bregma was marked and a hole was drilled through the bone at the coordinates of the VTA according to the atlas of Paxinos and Watson (Paxinos and Watson, 2007; anteroposterior -4.5 to $-5.7 \mathrm{~mm}$ to Bregma, lateral $0.3-1.2 \mathrm{~mm}, 7.2-9.5 \mathrm{~mm}$ below the cortical surface). Electrodes were manufactured in house from borosilicate capillaries (1.5 mm, Harvard Apparatus Limited, UK) pulled on a PP-830 electrode puller (Narishige, Japan) and filled with an electrolyte solution $(\mathrm{NaCl} 147 \mathrm{mM}$, adjusted to $\mathrm{pH} 6$ with $\mathrm{NaOH}$ ). The tip of the electrode was broken down under a microscope to an external diameter of 1-1.5 $\mu \mathrm{m}$. Typical resistance was in the range 4-8 $\mathrm{M} \Omega$. Outputs from the electrode were sent to a Neurolog AC pre-amplifier and amplifier (Digitimer, UK). Signals were filtered and sent to an audio amplifier, a Tektronix 2201 digital storage oscilloscope, and a 1,401 interface connected to a computer running Spike 2 v5.21 (CED, Cambridge, UK) for data capture and analysis. The descent of the electrode was carried out using a hydraulic micromanipulator (Narishige). At the end of the experimental procedures, animals were euthanized by intravenous chloral hydrate overdose. Brains were dissected out and cut using a cryostat (Leica) to visually confirm track locations within the VTA. Neurons located outside of the VTA were excluded from the present study. During some recordings, pharmacological agents (apomorphine $20-70 \mu \mathrm{g} / \mathrm{kg}$, quinpirole $20 \mu \mathrm{g} / \mathrm{kg}$, methylphenidate $2-4 \mathrm{mg} / \mathrm{kg}$ or eticlopride $0.2 \mathrm{mg} / \mathrm{kg}$ ) were applied intravenously via the lateral vein tail catheter to test for firing rate inhibition through dopamine $\mathrm{D}_{2}$ autoreceptor negative feedback (Federici et al., 2005; Di Miceli et al., 2019).

\section{Data Analysis and Statistics}

The mean basal firing activity was evaluated during at least $5 \mathrm{~min}$, after achieving a stable firing rate. Burst activities were 
detected using a standard protocol (Grace and Bunney, 1984), where at least two spikes are occurring within an interval of $80 \mathrm{~ms}$ or less, followed by a silence period of at least $160 \mathrm{~ms}$. For each neuron, burst activities are expressed as the percentage of all spikes occurring in bursts. Firing regularity was assessed by calculating coefficients of variation (CV) of the firing rates (standard deviation divided by mean). Both principal component analyses (PCA) and hierarchical cluster analyses were performed using R free software (R Core Team, 2013). PCA analysis was carried out to identify the parameters accounting for variability in our data set, as performed in another study (Mao et al., 2019), using the prcomp function in R (R Core Team, 2013; Jolliffe and Cadima, 2016) and drawn with the ggbiplot function $(\mathrm{Vu}, 2011)$. Raw data were analyzed using singular value decomposition of the scaled data matrix. More specifically, for each electrophysiological property, the dataset (matrix) was scaled from 0 to 1 to normalize (scaling) all values. Then, to adapt our scaled data matrix to multivariate analyses, such as PCA, the data was subsequently transformed using singular value decomposition, which performs linear transformations of a matrix. Such a process is a fundamental mathematical theorem that was explained previously (Golub and Van Loan, 1996). These transformations are applied directly with the aforementioned $\mathrm{R}$ functions. We used a total of six variables. These six variables were: (1) firing rate (i.e., the number of spikes generated in $1 \mathrm{~s})$; (2) CV of firing rates (firing regularity index); (3) bursting activity (i.e., the percentage of all spikes included in bursting events); (4) AP duration (i.e., the total AP duration; (5) $\Delta \mathrm{t}_{1}$ duration (i.e., the time from initiation of depolarization until end of repolarization; as well as (6) notched AP waveforms (i.e., the presence or not of a notch). Then, we determined the optimal number of clusters within our data set by applying a silhouette analysis (Rousseeuw, 1987; Charrad et al., 2014; Mao et al., 2019), using the Nbclust function in R (R Core Team, 2013). Such an analysis is designed to determine optimal coherence amongst values. This method measures the quality of clustering by calculating the Euclidean distances between identified clusters and calculates the optimal number of clusters to achieve ideal separation between all values, based on an array computation of 30 indices (Charrad et al., 2014). Finally, raw data was plotted using hierarchical analysis with Ward's method (Ward, 1963), based on the ideal cluster number found following the silhouette analysis. The resulting cluster dendrogram was generated using eclust and fviz_dend functions (Kassambara and Mundt, 2017). Results are expressed as the mean \pm standard error of the mean (SEM). On box-plots (boxand-whiskers), means are represented by a cross $(+)$, together with the $5-95 \%$ percentile range. Outliers out of the 5-95\% percentile were plotted individually but were not excluded from all analyses. For comparison between three groups, the Kruskal-Wallis one-way analysis of variance (ANOVA) tests were performed, followed by Dunn's post hoc tests. For contingency analyses, chi-square tests were used. For all tests, statistical significance was considered if $p<0.05$. All statistical tests were performed with GraphPad Prism 7.0 (GraphPad Software Inc., San Diego, CA, USA). All graphs were drawn using GraphPad, except silhouettes, PCA and dendrograms, which were drawn using R (R Core Team, 2013). Graphs were designed using colours optimized for the color-blind (Cox, 2015).

\section{RESULTS}

\section{Dataset Characteristics}

Three-hundred and eleven neurons were initially recorded in 92 animals. Twenty neurons were excluded a posteriori due to unstable recordings. Thus, our dataset included 291 neuronal recordings in the VTA using in vivo extracellular electrophysiology. Positions of the recorded neurons were confirmed at the end of the experiments using stereotaxic coordinates and track positions. Examples of some recorded neuron positions are drawn in Figure 1A. Spontaneouslyactive neurons were recorded during electrode descents $(n=2.3 \pm 0.2$ neurons/descent; Figure 1B). Burst activity was detected using a standard protocol for DA neurons (Grace and Bunney, 1984), where at least two spikes are occurring within an interval of $80 \mathrm{~ms}$ or less, followed by a silence period of at least $160 \mathrm{~ms}$ (Figure 1C). Across our data set, firing rates were log-normally distributed (Figures 1D1,D2,E), as previously observed elsewhere in the central nervous system (Mizuseki and Buzsáki, 2013; Buzsáki and Mizuseki, 2014; Petersen and Berg, 2016). Normal distributions were also observed for AP and $\Delta t_{1}$ durations (Figures 1F,G, respectively). We also observed a linear relationship between AP and $\Delta t_{1}$ durations (Figure $\mathbf{1 H}$ ). Bursting activities were not normally distributed since half of the neurons did not burst (145/291). In the remaining 146 neurons that did burst, bursting activities were not normally distributed. However, following logarithmic transformations, bursting activities presented log-normal distributions (Supplementary Figure S1). Neuronal firing regularity was assessed by calculating the $\mathrm{CV}$ from firing rates. Interspike interval distributions are presented as examples in Figures 1I,J. A typical recording example is provided in Figure 1K, showing stable firing rates over time.

\section{Hierarchical Clustering of VTA Neurons}

Overall, all the electrophysiological properties were largely variable among the recorded neurons and did not allow easy distinction between non-overlapping populations when considering their distributions. To identify subpopulations of putative DA neurons, we first excluded from the dataset putative GABAergic neurons, as defined by their firing frequency above $10 \mathrm{~Hz}$, which is consistently observed in the VTA (Steffensen et al., 1998; Luo et al., 2008; Li et al., 2012). In the present study, 22 neurons fired at rates over $10 \mathrm{~Hz}$ (Figure 2A). Hereafter, we considered these neurons as belonging to "cluster 1."

On the remaining 269 neurons, we used several electrophysiological criteria to perform clustering within our dataset: (1) firing rate; (2) firing rate CV (firing regularity index); (3) bursting activity (i.e., the percentage of spikes in burst); (4) AP durations; (5) $\Delta t_{1}$ (i.e time from the start of the depolarization to the end of the repolarization) durations as well as the (6) presence of a notched AP waveform. Using 
A

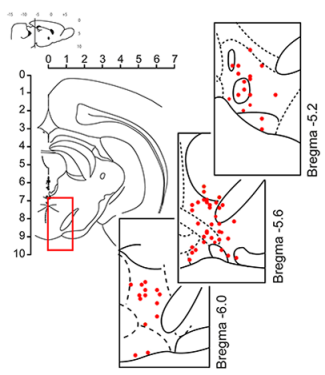

D1

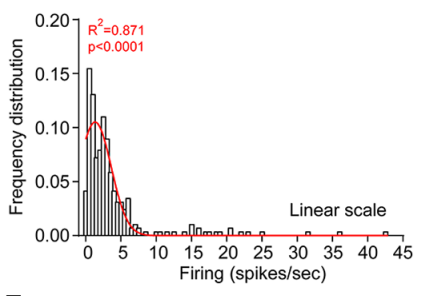

$\mathbf{F}$

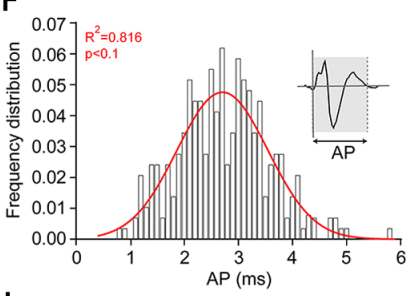

I

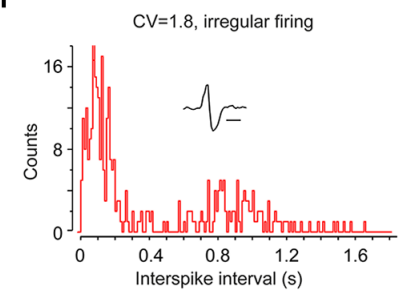

B

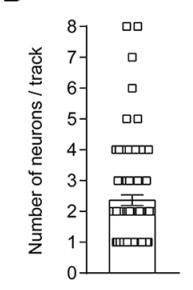

C

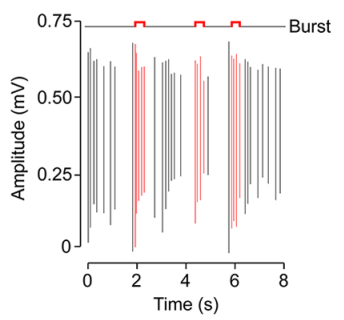

E
D2

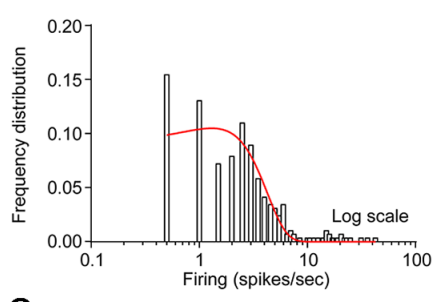

G

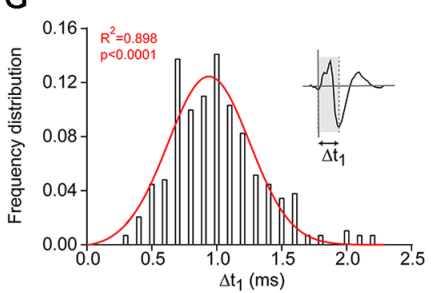

$\mathbf{J}$

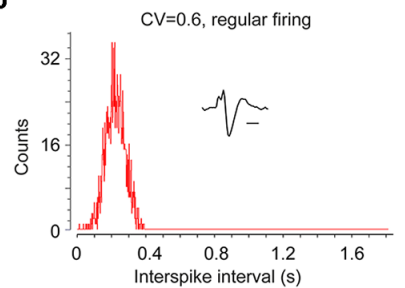

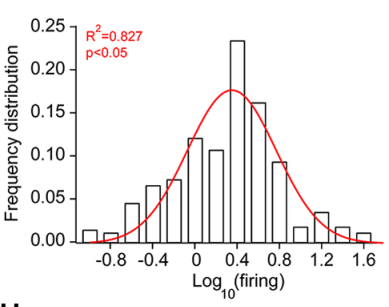

H

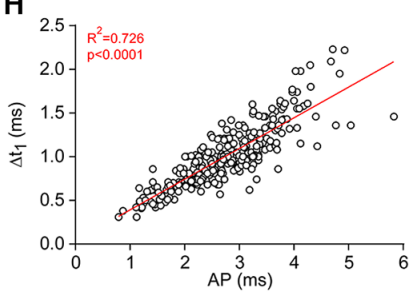

K
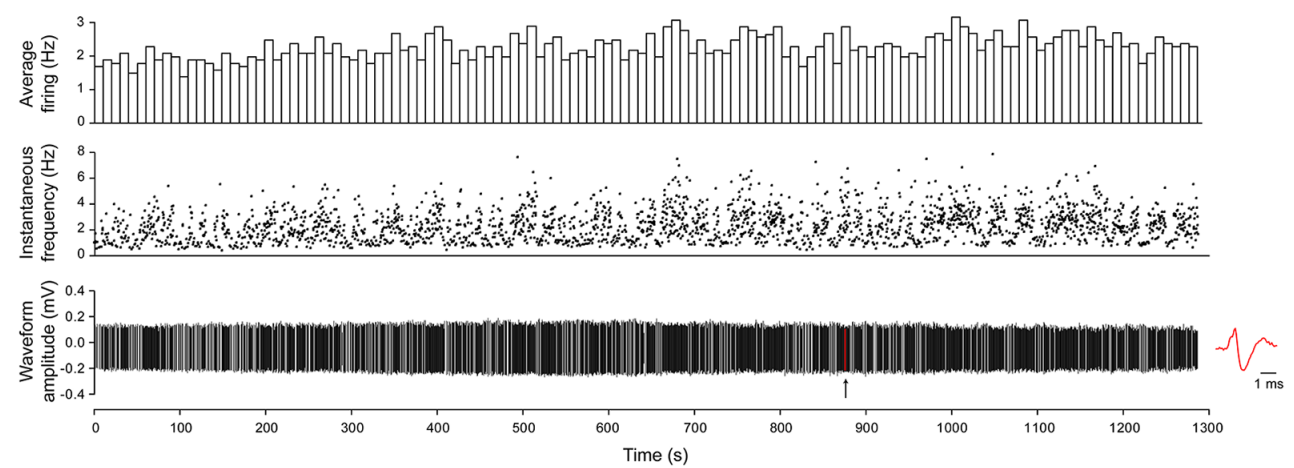

FIGURE 1 | Dataset acquired using in vivo extracellular recordings of ventral tegmental area (VTA) neurons in anesthetized rats. (A) VTA location on a rat coronal brain section, adapted from Paxinos and Watson (2007). Insets: examples of coordinate locations of some neurons, reconstructed from stereotaxic measurements. All units are in mm. (B) Number of spontaneously-active neurons encountered during electrode descents. (C) Representative example of burst activities during in vivo extracellular electrophysiology. Red action potential (AP) waveforms are included within burst events while black AP waveforms are outside of burst events. Firing-rate distributions using linear (D1) or logarithmic (D2) scales. Firing-rate logarithmic values (E). Red curves represent fitted Gaussian curves. Note that firing rates are skewed (asymmetric Gaussian, in D1,D2) but also lognormally-distributed (symmetric Gaussian, in E). Across the 291 neurons, AP (F) and $\Delta t_{1}$ (G) durations were normally distributed. The direct linear relationship between AP and $\Delta t_{1}$ durations $\mathbf{( H )}$. Representative inter-spike interval distributions and AP waveforms of regularly (I) and irregularly $\mathbf{( J )}$ firing neurons. The horizontal bars represent $1 \mathrm{~ms}$. Note the differences in coefficient of variation (CV; firing rate coefficient of variation). (K) Typical recording example of VTA dopaminergic (DA) neurons, displaying a stable firing rate over time. The arrow indicates the location of the AP waveform represented on the right. 

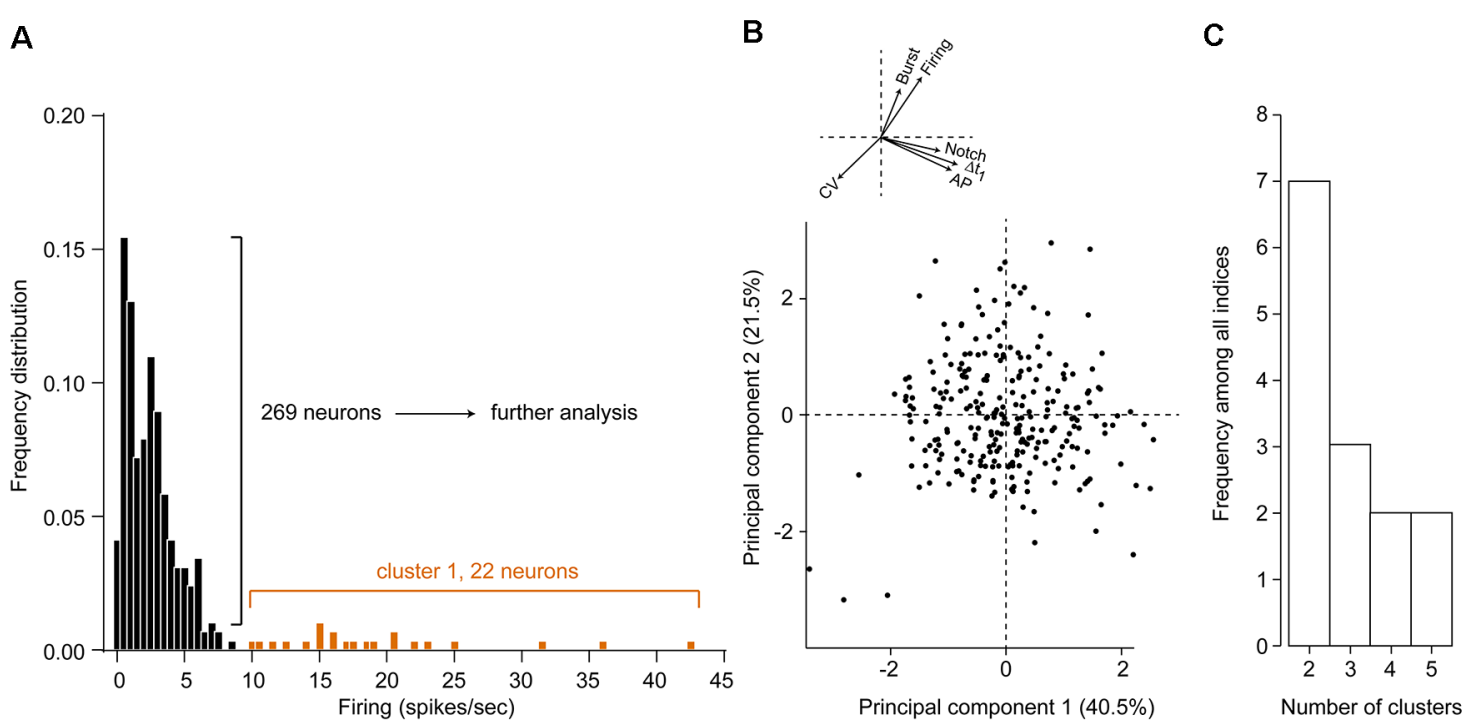

D

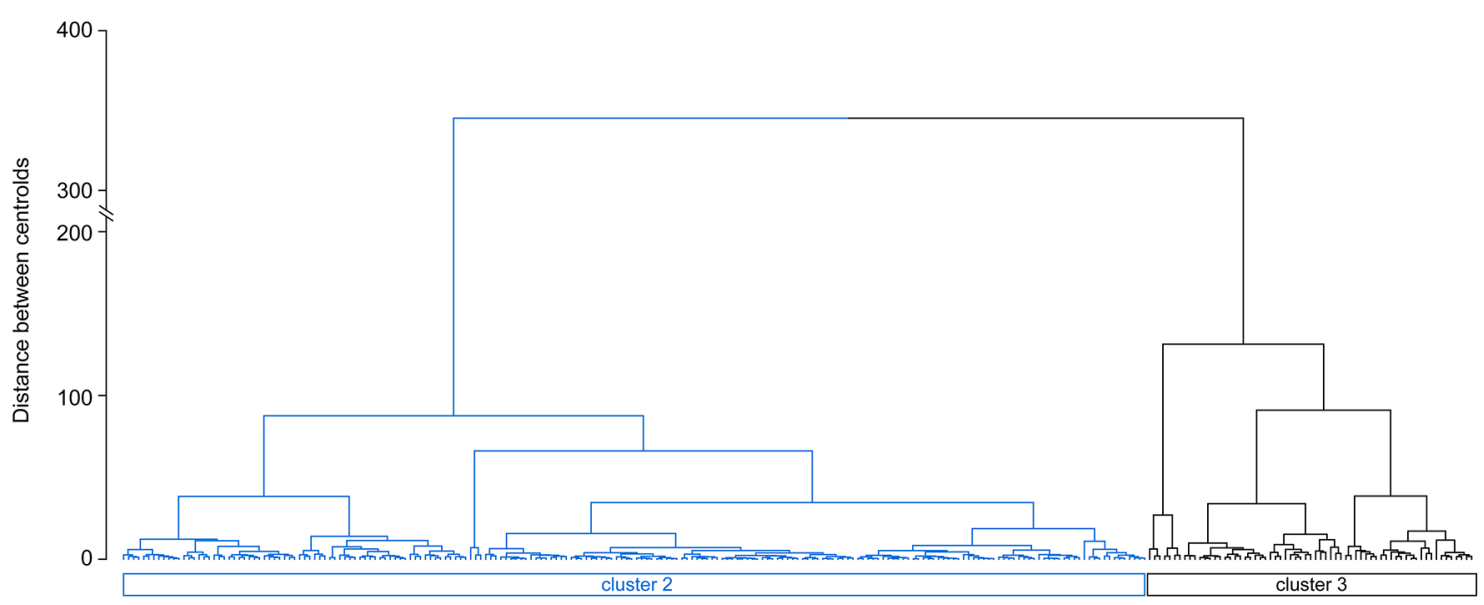

E

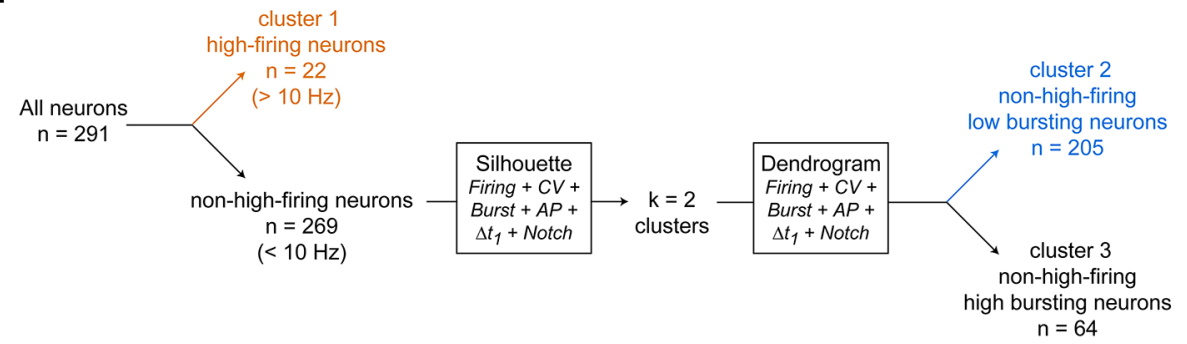

FIGURE 2 | Unsupervised clustering of the 269 non-high-firing neurons. (A) Initial segregation of neurons with firing rates above $10 \mathrm{~Hz}$. (B) Principal component analysis (PCA) using all six electrophysiological variables revealed that the first two principal components accounted for $62 \%$ of the total variance within the dataset. Arrows indicate vectors of the six variables. For clarity, these arrows and their respective variables are drawn again at the top left. (C) Silhouette analysis determined that the optimal number of clusters within our dataset is 2. (D) Unsupervised agglomerative hierarchical clustering with two clusters, using Euclidean distances and Ward's method for optimal branching. (E) Methodology flow chart used in the present study.

principal component analysis (PCA) on the scaled data matrix of these six electrophysiological variables, we found that $62 \%$ of total variability could be captured by the first two principal components (Figure 2B), which represents successful variance capture within the PCA analysis and adequate reduction of dimensionality. Amongst the six parameters, none could be ruled out for not inducing variance, as observed in the vectors drawn in Figure $\mathbf{2 B}$. Therefore, all six parameters 
were subsequently used to cluster neurons. Using silhouette analysis, which determines separation distance between the resulting clusters and between each neuron, segregation of these 269 neurons was found to be efficient using two clusters, as two clusters are the most frequent ones to be output by the indices with the silhouette analysis (Figure 2C). Subsequently, hierarchical agglomerative clustering (Ward's method) was drawn using two clusters based on these six electrophysiological properties (Figure 2D). These two clusters ("cluster 2" and "cluster 3") segregated two different subpopulations of VTA neurons, based on optimal branching between each neuron within the entire dataset. A flow chart for the successive steps performed herein is given in Figure 2E.

\section{Electrophysiological Properties of Clustered VTA Neuronal Populations}

As defined by the criteria used to isolate cluster 1 (firing rate $>10 \mathrm{~Hz}$ ), neurons from cluster 1 displayed significantly higher firing rates than neurons within cluster 2 or 3 . Moreover, firing rates from neurons within cluster 2 were also significantly lower than neurons in cluster 3 (cluster 1: $19.47 \pm 1.76$ spikes/s, cluster 2: $2.10 \pm 0.12$ spikes/s, cluster 3: $3.32 \pm 0.23$ spikes/s, $p<0.001$, Kruskal-Wallis one-way ANOVA, Figure 3A, Table 1). Bursting activities (defined for each neuron as the percentage of all spikes occurring in burst events) were significantly higher in neurons from cluster 3 compared to neurons from either cluster 1 or 2 (cluster 1: $0.07 \pm 0.07 \%$, cluster 2: $3.29 \pm 0.36 \%$, cluster 3: $37.97 \pm 1.91 \%, p<0.001$, Kruskal-Wallis one-way ANOVA, Figure 3B, Table 1). When representing firing rates according to burst activities, we observed that neurons from each cluster formed distinguishable groups (Figure 3C).

Similarly, as expected, both AP and $\Delta \mathrm{t}_{1}$ durations were significantly shorter in neurons from cluster 1 compared to neurons from either cluster 2 or 3 [(AP durations) cluster 1: $1.54 \pm 0.08 \mathrm{~ms}$, cluster 2: $2.79 \pm 0.05 \mathrm{~ms}$, cluster 3 : $3.00 \pm 0.10 \mathrm{~ms}, p<0.001$, Kruskal-Wallis one-way ANOVA, Figure 3D and Table 1; $\left(\Delta \mathrm{t}_{1}\right.$ durations) cluster 1: $0.60 \pm 0.03 \mathrm{~ms}$, cluster 2: $1.01 \pm 0.02 \mathrm{~ms}$, cluster 3: $1.11 \pm 0.05 \mathrm{~ms}, p<0.001$, Kruskal-Wallis one-way ANOVA, Figure $3 \mathrm{E}$ and Table 1]. However, neurons from clusters 2 and 3 were not different for $\mathrm{AP}$ and $\Delta \mathrm{t}_{1}$ durations.

The presence of notch was rarely observed (1/22) in neurons belonging to cluster 1 (Figure 3F and Table 1). Remarkably, in clusters 2 and 3, half of all neurons presented notched AP waveforms (respectively 102/205 and 34/64 neurons, $p=0.64$, chi-square test).

Some neurons (107/291), but not all, were tested for pharmacological response to dopamine $\mathrm{D}_{2}$ autoreceptor activation, by intravenously injecting apomorphine (20-70 $\mu \mathrm{g} / \mathrm{kg}, n=15)$, quinpirole $(20 \mu \mathrm{g} / \mathrm{kg}, n=26)$ or methylphenidate $(2-4 \mathrm{mg} / \mathrm{kg}, n=66)$. These neurons were found to be clustered in both clusters 2 and 3 (Figure 3G).

It is also worth noticing that, amongst all neurons, AP waveform heterogeneity was high (Figure $\mathbf{3 H}$ ), as observed and explained by others (López-Jury et al., 2018). Indeed, notch amplitude and position occurrence (during the rise or fall of the AP) varied largely across all neurons. This indicates that the presence of a notched AP might not be an optimal discriminating factor. A typical pharmacological recording is illustrated in Figure 3I.

\section{DISCUSSION}

As for several brain regions, it is now clear that the VTA contains subpopulations of DA neurons. Thus, it remains crucial to differentiate these neuronal subpopulations when studying VTA neural circuits. Electrophysiological studies of VTA neurons often rely on a combination of parameters to identify the neuronal population to which they belong. Such an approach can lead to bias classification, as these parameters are usually not mutually exclusive. Moreover, several characteristics may overlap in different populations, at least concerning electrophysiological properties (Margolis et al., 2006). Here, we observed that initial sorting with firing rates above $10 \mathrm{~Hz}$ followed by the combination of the six classical electrophysiological parameters used in the field (firing rate and its regularity, bursting activity, $\mathrm{AP}$ and $\Delta \mathrm{t}_{1}$ durations, presence of AP notched waveform) into an objective hierarchical clustering resulted in the segregation of VTA neurons into three groups (Figure 2), whose characteristics are summarized in Table 1 . The present study also highlights the fact that burst activities can segregate two populations with high-bursting ( $>17.5 \%$ of spikes in bursts) from low-bursting neurons $(<20 \%$ of spikes in bursts) within non-high-firing neurons.

We used a cut-off criterion at $10 \mathrm{~Hz}$ to separate high-firing neurons from the remaining non-high-firing neurons, according to previous studies showing that GABAergic neurons fire above $10 \mathrm{~Hz}$ in anesthetized (Yim and Mogenson, 1980; Steffensen et al., 1998, 2008, 2009; Stobbs et al., 2004) and freely behaving animals (Gallegos et al., 1999; Liu et al., 2012). In these neurons, we found almost no bursting activities, which is consistent to another study, using the same recording technique (Steffensen et al., 1998). Therefore, cluster 1 represents the smallest proportion of neurons (7.6\%), likely GABAergic, which are characterized by very high firing rates and almost no bursting activities, together with short AP and $\Delta \mathrm{t}_{1}$ durations. Regular firing discharges were observed in these neurons, but they did not exhibit notched AP waveforms, except in one neuron (Figure 3, Table 1). However, compared to immunohistochemistry studies, we found relatively lower proportions of GABAergic neurons (7.6\%), compared to what was previously described (20-30\%). This can be explained by a selection bias in part of the experiments, to focus on non-high-firing neurons (rates below $10 \mathrm{~Hz}$ ).

Within non-high-firing neurons, clusters 2 and 3 engulfed $70.4 \%$ and $22 \%$ of all recorded neurons, respectively. These neurons are characterized by firing rates between $2-3.6 \mathrm{~Hz}$ (Table 1). Previous studies have shown that DA neurons present a mean firing rate of $4 \mathrm{~Hz}$ in anesthetized (Yim and Mogenson, 1980; Sanghera et al., 1984; Trulson and Trulson, 1987; Clark and Chiodo, 1988; Marinelli and White, 2000; Brandon et al., 2003; Ungless et al., 2004; Bennett and Gronier, 2007; Luo et al., 2008; Chernoloz et al., 2009; Valenti and Grace, 2010; 

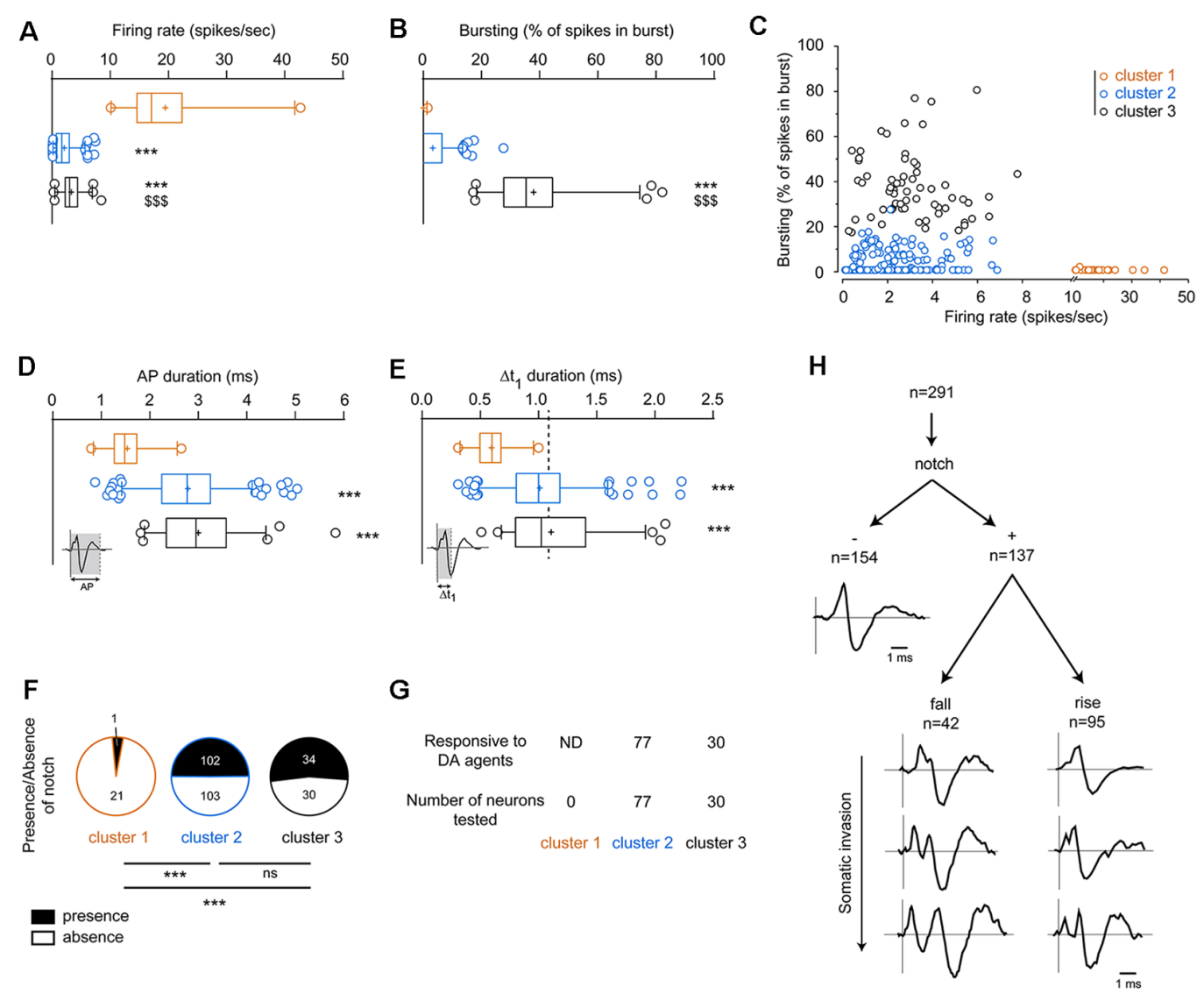

G

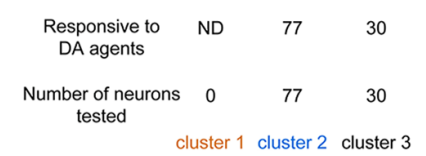

$1 \mathrm{~ms}$
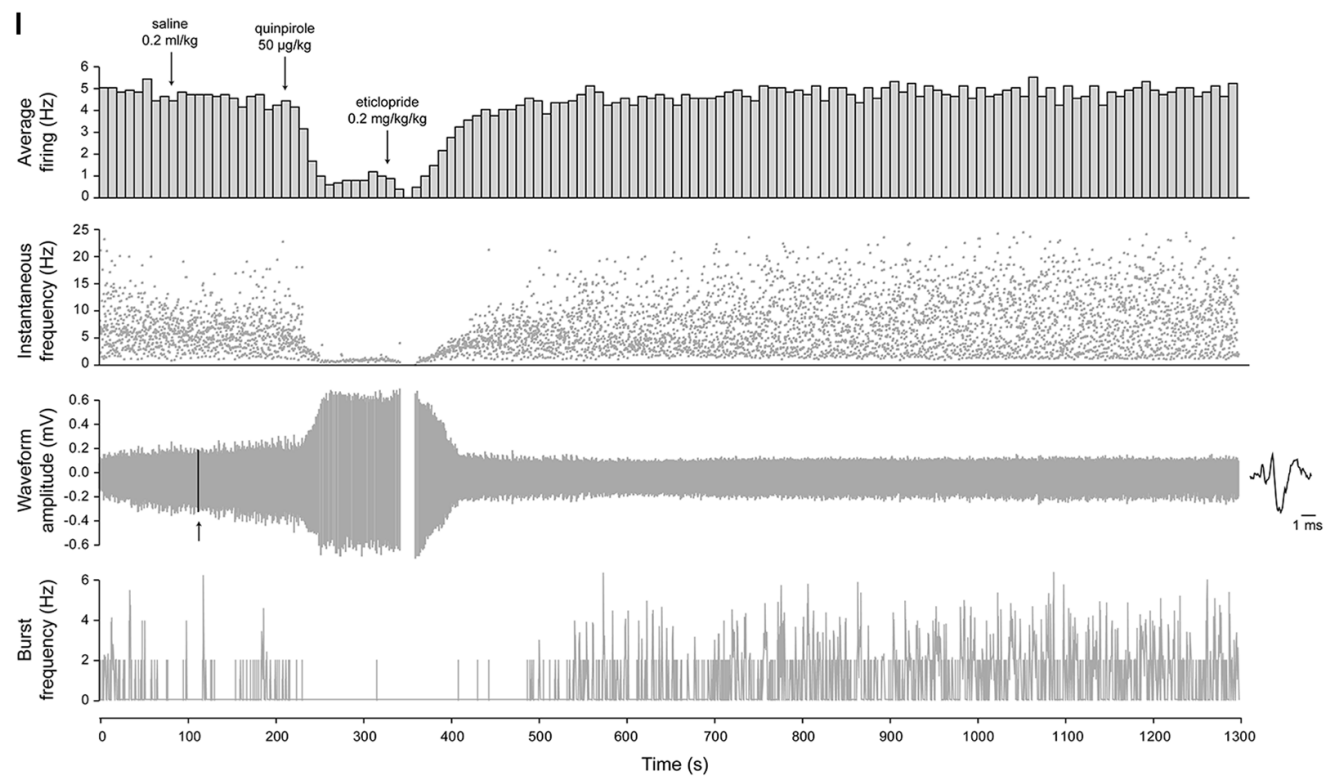

FIGURE 3 | Segregation of VTA neuronal populations according to their electrophysiological properties. (A) Firing rates are significantly higher in cluster 1 than clusters 2 and 3 . Neurons from cluster 2 displayed significantly lower firing rates than neurons from cluster 3 . (B) Neurons from cluster 3 displayed significantly greater bursting activities than neurons from either cluster 2 or 3. (C) Firing rates and bursting activities in neurons from each cluster. High-firing neurons belonging to cluster 1 presented very minimal bursting activities. Generally, neurons belonging to cluster $3(n=64)$ presented burst activities above $17.5 \%$ of all spikes in bursts, while neurons belonging to cluster $2(n=205)$ displayed burst activities below $17.5 \%$. Here, these three clusters are segregated. (D) Total action potential (AP) durations are significantly lower in the high-firing neurons (cluster 1) than in neurons from cluster 2 or 3 . (E) Similarly, $\Delta t_{1}$ durations from cluster 1 were significantly lower than cluster 2 or 3 . The dashed line represents the well-established cut-off criterion to distinguish between DA and non-DA neurons at 1.1 ms (Ungless and Grace, 2012). 


\section{FIGURE 3 | Continued}

(F) Presence/absence of notched AP waveforms in neurons belonging to clusters 1, 2, or 3. Statistical results were performed using Chi-square tests. (G) Neurons responsive to DA pharmacology (apomorphine 10-70 $\mu \mathrm{g} / \mathrm{kg}$, quinpirole 20-60 $\mu \mathrm{g} / \mathrm{kg}$ or methylphenidate $2-4 \mathrm{mg} / \mathrm{kg}$ ) were found in both cluster 2 and 3 . Note that some neurons were not tested. (H) Within all neurons included in the present study $(n=291)$, great heterogeneity of AP waveforms was observed. One-way Kruskal-Wallis analysis of variance (ANOVA) results are given as following: ${ }^{* \star *} p<0.001$ vs. cluster 1 and $\$ \$ p<0.001$ vs. cluster 2. (I) Typical recording example of VTA neurons where the dopamine $D_{2}$ receptor agonist quinpirole $(50 \mu \mathrm{g} / \mathrm{kg}, i \mathrm{i})$ induces autoreceptor-mediated negative feedback, which is reversed by the dopamine $D_{2}$ receptor antagonist eticlopride $(0.2 \mathrm{mg} / \mathrm{kg}, \mathrm{iv})$. Note that quinpirole greatly increased the AP amplitude. The arrow indicates the location of the AP waveform represented on the right. All of the histograms represented here are box-plots (box and whiskers). The "+" sign represents the mean. The horizontal bar represents the median value. The interquartile range (Q1-Q3) is displayed with a vertical rectangle ("box"), while the thin lines ("whiskers") represent the intervals between the lowest/highest values and the Q1/Q3 quartiles, respectively. Outlying values are represented individually with open circles but were not excluded from all analyses. Asterisks ${\left({ }^{\star \star \star}\right)}$ are used to indicate significantly different values vs. cluster 1 , while dollars $(\$ \$$ ) are used to indicate significantly different values vs. cluster 2 . ns: non-significant.

Valenti et al., 2012; Oosterhof et al., 2014, 2015; El Iskandrani et al., 2015) and freely behaving animals (Hyland et al., 2002; Shen, 2003; Dahan et al., 2007; Li et al., 2012). When these neurons are recorded using ex vivo patch-clamp electrophysiology, lower mean firing rates are reported, around $1 \mathrm{~Hz}$ (Federici et al., 2005), likely due to altered circuitry dynamics induced by the slicing procedure.

When focusing on bursting activities, we found in the present study that neurons belonging to cluster 3 presented higher bursting activities (38\%, Table 1) compared to neurons engulfed in cluster 2 (3\%, Table 1). According to previous research, bursting activities of VTA DA neurons are included within the $20-40 \%$ range in anesthetized (Clark and Chiodo, 1988; Marinelli and White, 2000; Brandon et al., 2003; Bennett and Gronier, 2007; Chernoloz et al., 2009; Valenti and Grace, 2010; Valenti et al., 2012; Oosterhof et al., 2015) and awake animals (Hyland et al., 2002; Dahan et al., 2007; Li et al., 2012), although a few studies reported different values (Marinelli and White, 2000; Shen, 2003). Interestingly, one study reported DA neurons with low bursting activities (Marinelli and White, 2000), likely reflected in cluster 2 in our study (Table 1).

In the present study, we observed that several electrophysiological properties are normally distributed when represented on logarithmic scales. Indeed, firing rates, AP and $\Delta \mathrm{t}_{1}$ durations (Figure $\mathbf{1}$ ) as well as bursting activities (Supplementary Figure S1) presented log-normal distributions across all neurons. Such a principle was previously observed with firing rates in spinal motor networks (Petersen and Berg, 2016) and hippocampal pyramidal neurons (Mizuseki and Buzsáki, 2013). This suggests that skewed distributions of quantitative variables are conserved across the brain region and that such processes arise from complex and robust neuronal network computation (Mizuseki and Buzsáki, 2013). These log-normal distributions are also observed outside of neuroscience (Limpert et al., 2001).

AP properties were not discriminatory factors among VTA neurons, compared to firing patterns. Indeed, a great overlap was observed in the two clusters for total AP duration and $\Delta t_{1}$ duration (clusters 2 and 3, Table 1). These characteristics are consistent with what has being described for VTA DA neurons (Ungless and Grace, 2012). Besides, the presence of a notched AP waveform is observed in around half of the neurons from both groups, showing that a certain heterogeneity exits. A recent study has explained that notched events are artifact electric event, due to the parameters surrounding the recording technique (López-Jury et al., 2018). Thus, even though AP properties are widely used to identify DA neurons, these electrophysiological parameters appear weak to segregate DA subpopulations in the VTA. The present study showed that AP durations and $\Delta t_{1}$ durations are directly proportional, indicating that measuring one or the other should be sufficient. Total AP durations of neurons within clusters 2 and 3 are within the $2.8-3 \mathrm{~ms}$ range (Table 1). Previous studies have reported AP durations of VTA DA neurons within the 2-3 ms range in anesthetized animals (Brandon et al., 2003; Luo et al., 2008; Chernoloz et al., 2009; Mejias-Aponte et al., 2015), although another study reported a 2-5 ms range during both in vivo and ex vivo recordings (Trulson and Trulson, 1987). With ex vivo patch-clamp electrophysiology, AP durations are often reported to greatly vary according to projection sites (Lammel et al., 2008; Margolis et al., 2008). In awake animals, a study reported AP duration over $2 \mathrm{~ms}$, with a mean at $3.5 \mathrm{~ms}$ (Dahan et al., 2007), while another study found shorter AP duration, around $1.5 \mathrm{~ms}$ (Liu et al., 2012). Finally, we also outline that non-DA neurons possess similar AP duration than DA neurons, as observed previously (Ungless et al., 2004), suggesting that AP duration is an overlapping sorting criterion.

In light with a previous study showing that AP waveforms during in vivo extracellular electrophysiology are strongly biased by pipette positioning and neuronal morphology (LópezJury et al., 2018), we suggest that AP properties should not solely be used to determine neuronal populations. An exception, however, should be very short AP waveforms, usually with total AP duration below $1 \mathrm{~ms}$, which are consistently reported as typical characteristics of GABAergic neurons using different in vivo techniques (Steffensen et al., 1998; Gallegos et al., 1999; Stobbs et al., 2004; Li et al., 2012; Liu et al., 2012). Notched AP waveforms are usually selected by investigators to distinguish DA from non-DA neurons during in vivo extracellular electrophysiology (Ungless and Grace, 2012). While this can represent bias, we also argue that such an approach would lead to the homogeneous neuronal selection, based on similar neuronal morphologies in these neurons (López-Jury et al., 2018). The hypothesis that notched AP waveforms are solely observed in DA neurons remains an open question, which should be carefully examined in future studies. However, we suggest that experimenters should not use such a criterion to distinguish DA from non-DA neurons.

In the present study, we injected DA agonists intravenously to observed neuronal responses. However, this approach was not 
TABLE 1 | Electrophysiological characteristics of VTA neuronal populations segregated by hierarchical clustering

\begin{tabular}{|c|c|c|c|}
\hline & Cluster 1 & Cluster 2 & Cluster 3 \\
\hline Firing rate $(\mathrm{Hz})$ & $19.47 \pm 1.76$ & $2.10 \pm 0.12$ & $3.32 \pm 0.23$ \\
\hline Burst activity (\%) & $0.07 \pm 0.07$ & $3.29 \pm 0.36$ & $37.97 \pm 1.91$ \\
\hline Presence of bursting activity & $n=1 / 22$ & $n=81 / 205$ & $n=64 / 64$ \\
\hline AP duration (ms) & $1.54 \pm 0.08$ & $2.79 \pm 0.05$ & $3.00 \pm 0.10$ \\
\hline$\Delta \mathrm{t}_{1}$ duration $(\mathrm{ms})$ & $0.60 \pm 0.03$ & $1.01 \pm 0.02$ & $1.11 \pm 0.05$ \\
\hline Presence of notch & $n=1 / 22$ & $n=102 / 205$ & $n=34 / 64$ \\
\hline Characteristics & High-firing & Non-high-firing Low/No bursting & Non-high-firing High bursting \\
\hline
\end{tabular}

systematically performed, as we aimed to classify VTA neurons according to electrophysiological properties only, which would be necessary for studies where pharmacological identification of VTA neurons would consist in an a priori alteration of neuronal properties. All of the neurons tested responded by firing rate decrease, together with decreased bursting activities (Figure 3I). These neurons were sorted in both clusters 2 and 3 , likely reflecting the fact that DA neurons are engulfed in these two clusters. The pharmacological responses of VTA DA neurons to DA agents have been extensively described in previous studies using in vivo extracellular electrophysiology in anesthetized animals, both from our laboratory (Bennett and Gronier, 2007; Di Miceli et al., 2019) as well as many others (Aghajanian and Bunney, 1977; Sanghera et al., 1984; Trulson and Trulson, 1987; Clark and Chiodo, 1988; Ackerman et al., 1993; Marinelli and White, 2000; Brandon et al., 2003; Guiard et al., 2008; Chernoloz et al., 2009; El Mansari et al., 2010; Valenti and Grace, 2010; Panin et al., 2012; Oosterhof et al., 2014, 2015). Similar responses were observed using ex vivo patch-clamp electrophysiology (Federici et al., 2005; Lammel et al., 2008; Margolis et al., 2008), although some specific subpopulations of DA neurons do not respond to such a pharmacological approach (Lammel et al., 2008; Margolis et al., 2008). Such a feature is also present in awake animals (Hyland et al., 2002; Li et al., 2012). However, non-DA neurons are also responding to such a paradigm, showing that response to DA pharmacology is shared amongst DA and non-DA neurons. Indeed, putative non-DA responded to DA agonists in anesthetized (Yim and Mogenson, 1980; Luo et al., 2008) and awake animals (Kiyatkin and Rebec, 1998), as well as in brain slices (Johnson and North, 1992; Cameron et al., 1997; Margolis et al., 2006). To note, some GABAergic neurons were found to be excited following exposure to DA agonists using extracellular in vivo recordings in anesthetized animals (Stobbs et al., 2004; Steffensen et al., 2008).

Beside GABAergic and DA neurons, the VTA also contains a small proportion of glutamatergic and glutamatergicdopaminergic co-expressing neurons. These neuronal populations are less described (Hnasko et al., 2012; Morales and Margolis, 2017; Root et al., 2018) and the proportion varies widely between studies, depending on their location in the VTA [from 2\% to more than 50\% (Yamaguchi et al., 2011)]. Thus, we cannot rule out that glutamate-expressing neurons could be present in our clusters 2 and 3. One study reported non-DA neurons (TH-negative) with firing rates below $2 \mathrm{~Hz}$ (Ungless et al., 2004), which could represent glutamatergic neurons.
The same study, however, also observed some DA neurons (TH-positive) with firing rates below $2 \mathrm{~Hz}$ (Ungless et al., 2004). Identification of either DA, GABAergic or glutamatergic neurons, using electrophysiology, immunohistochemistry, RT-PCR or optogenetics, remains equivocal (Chiodo et al., 1984; Margolis et al., 2006, 2008; Luo et al., 2008; Morales and Root, 2014; Yamaguchi et al., 2015; Morales and Margolis, 2017). Heterogeneity of VTA DA neurons could also be explained by different afferent glutamatergic inputs (Margolis et al., 2005), which we could not evaluate in the present study. To precisely parallel electrophysiological properties with the identity of each neuron, challenging experiments such as juxtacellular recording with iontophoresis (Pinault, 1996) or in vivo patch-clamp recordings followed by single-cell RT-PCR would be needed.

Our two groups, clusters 2 and 3, likely reflect the wide distribution of bursting activities found in DA neurons. Indeed, when identifying DA neurons, the criteria for determining bursting activities remain vague (Grace and Bunney, 1984; Ungless and Grace, 2012). Hence, our study highlights the possible relevance of bursting activity in segregating DA subpopulations in the VTA. We acknowledge that a few parameters, such as anesthesia, can alter the electrophysiological properties of VTA DA neurons, as demonstrated by previous studies (Appel et al., 2006; Marinelli and McCutcheon, 2014). Indeed, ketamine-based anesthetics are known to alter NMDA neurotransmission (Moghaddam et al., 1997) and thus the firing properties of several types of neurons (Björkholm et al., 2015; El Iskandrani et al., 2015). On the other hand, urethane can alter respiratory parameters in rodents (Hughes et al., 1982), although we observed similar electrophysiological properties between the present study and another study obtained in urethane-anesthetized animals. To control for possible electrophysiological variability, all experiments in the present study were performed under similar conditions, using chloral hydrate. Besides, numerous studies used such an anesthetic to describe the electrophysiological properties of VTA DA neurons. Comparisons between different studies, however, should be used with caution, as a few electrophysiological properties of VTA DA neurons can vary according to the recording techniques used (Sanghera et al., 1984).

To conclude, we confirm in the present study that the VTA harbors heterogeneous neuronal populations, based on hierarchical clustering analysis, at least concerning electrophysiological properties. This work highlighted the fact that firing pattern analyses cluster different subclasses of DA neurons, while AP characteristics (width and shape) 
are less likely to reflect such segregation. The clustering of neurons in the present study was based on electrophysiological properties, which can reflect differential excitability depending on the different inputs/outputs functions of the neuronal subpopulations found within the VTA. Finally, we suggest that only short AP duration should be used to rule out non-DA neurons within the VTA since such a trait appears as characteristic of GABAergic neurons, as well as firing rates above $10 \mathrm{~Hz}$. Moreover, experimenters should not discard low-bursting neurons.

\section{DATA AVAILABILITY STATEMENT}

The datasets generated for this study are available on request to the corresponding author.

\section{ETHICS STATEMENT}

The animal study was reviewed and approved by De Montfort University.

\section{AUTHOR CONTRIBUTIONS}

$\mathrm{MD}, \mathrm{ZH}$, and $\mathrm{BG}$ : experimental design. $\mathrm{MD}$ and $\mathrm{BG}$ : data acquisition. $\mathrm{MD}, \mathrm{ZH}, \mathrm{PR}$, and $\mathrm{CB}-\mathrm{B}$ : data analysis. $\mathrm{MD}, \mathrm{ZH}$, $\mathrm{CB}-\mathrm{B}$, and XF: data curation. DC, SL, and BG: supervisions. DC, $\mathrm{SL}, \mathrm{XF}$, and BG: funding. MD and $\mathrm{ZH}$ : manuscript drafting and writing. All authors: critical analysis.

\section{REFERENCES}

Ackerman, J. M., Johansen, P. A., Clark, D., and White, F. J. (1993). Electrophysiological effects of putative autoreceptor-selective dopamine agonists on A10 dopamine neurons. J. Pharmacol. Exp. Ther. 265, 963-970.

Aghajanian, G. K., and Bunney, B. S. (1977). Dopamine"autoreceptors": pharmacological characterization by microiontophoretic single cell recording studies. Naunyn. Schmiedebergs Arch. Pharmacol. 297, 1-7. doi: 10.1007/bf00508803

Appel, S. B., Wise, L., McDaid, J., Koyama, S., McElvain, M. A., and Brodie, M. S. (2006). The effects of long chain-length $n$-alcohols on the firing frequency of dopaminergic neurons of the ventral tegmental area. J. Pharmacol. Exp. Ther. 318, 1137-1145. doi: 10.1124/jpet.106.105148

Beier, K. T., Steinberg, E. E., DeLoach, K. E., Xie, S., Miyamichi, K., Schwarz, L., et al. (2015). Circuit architecture of VTA dopamine neurons revealed by systematic input-output mapping. Cell 162, 622-634. doi: 10.1016/j.cell.2015. 07.015

Bennett, S., and Gronier, B. (2007). Effects of antipsychotic treatments and D-serine supplementation on the electrophysiological activation of midbrain dopamine neurons induced by the noncompetitive NMDA antagonist MK 801 . Synapse 61, 679-688. doi: 10.1002/syn.20413

Björkholm, C., Jardemark, K., Schilström, B., and Svensson, T. H. (2015). Ketamine-like effects of a combination of olanzapine and fluoxetine on AMPA and NMDA receptor-mediated transmission in the medial prefrontal cortex of the rat. Eur. Neuropsychopharmacol. 25, 1842-1847. doi: 10.1016/j.euroneuro. 2015.07.002

Brandon, C. L., Marinelli, M., and White, F. J. (2003). Adolescent exposure to methylphenidate alters the activity of rat midbrain dopamine neurons. Biol. Psychiatry 54, 1338-1344. doi: 10.1016/s0006-3223(03) 00787-x

\section{FUNDING}

This work was supported by an internal grant from De Montfort University given to MD. $\mathrm{ZH}$ was funded by Labex Brain (ANR-10-LABX-43, to DC and XF) and Agence Nationale de la Recherche (ANR; ANR-18-CE14-0029-02, to DC). MD is currently funded by the Fondation pour la Recherche Médicale (équipe FRM DEQ20170336724, to SL).

\section{ACKNOWLEDGMENTS}

We thank all the members from the NutriNeuro Laboratory and the Pharmacology and Neuroscience Research Group at De Montfort University for their useful discussions, the staff in the animal facilities for their daily help (Mrs. Anita O’Donoghue and Mr. Stephen Bowen ${ }^{\dagger}$ ), as well as all the persons involved in administrative support. The authors would also like to thank the Fondation pour la Recherche Médicale (FRM) for their financial supports. († in memoriam).

\section{SUPPLEMENTARY MATERIAL}

The Supplementary Material for this article can be found online at: https://www.frontiersin.org/articles/10.3389/fncir.2020.000 51/full\#supplementary-material.

FIGURE S1 | Bursting events across all neurons. (A) Frequency of bursting activities in all neurons from the dataset. (B) Frequency of bursting activities in bursting neurons only. (C) Bursting activities logarithmic values in bursting neurons.

Buzsáki, G., and Mizuseki, K. (2014). The log-dynamic brain: how skewed distributions affect network operations. Nat. Rev. Neurosci. 15, 264-278. doi: $10.1038 / \mathrm{nrn} 3687$

Cameron, D. L., Wessendorf, M. W., and Williams, J. T. (1997). A subset of ventral tegmental area neurons is inhibited by dopamine, 5-hydroxytryptamine and opioids. Neuroscience 77, 155-166. doi: 10.1016/s0306-4522(96)00444-7

Charrad, M., Ghazzali, N., Boiteau, V., and Niknafs, A. (2014). NbClust: an R package for determining the relevant number of clusters in a data set. J. Stat. Softw. 61:6. doi: 10.18637/jss.v061.i06

Chernoloz, O., El Mansari, M., and Blier, P. (2009). Sustained administration of pramipexole modifies the spontaneous firing of dopamine, norepinephrine, and serotonin neurons in the rat brain. Neuropsychopharmacology 34, 651-661. doi: 10.1038/npp.2008.114

Chiodo, L. A., Bannon, M. J., Grace, A. A., Roth, R. H., and Bunney, B. S. (1984) Evidence for the absence of impulse-regulating somatodendritic and synthesismodulating nerve terminal autoreceptors on subpopulations of mesocortical dopamine neurons. Neuroscience 12, 1-16. doi: 10.1016/0306-4522(84)90133-7

Clark, D., and Chiodo, L. A. (1988). Electrophysiological and pharmacological characterization of identified nigrostriatal and mesoaccumbens dopamine neurons in the rat. Synapse 2, 474-485. doi: 10.1002/syn.890020503

Cox, L. (2015). Tips for Designing Scientific Figures for Color Blind Readers. somersault18:24. Available online at: https://www.somersault1824.com/. Accessed July 1, 2020.

Dahan, L., Astier, B., Vautrelle, N., Urbain, N., Kocsis, B., and Chouvet, G. (2007). Prominent burst firing of dopaminergic neurons in the ventral tegmental area during paradoxical sleep. Neuropsychopharmacology 32, 1232-1241. doi: 10.1038/sj.npp.1301251

Di Miceli, M., Omoloye, A., and Gronier, B. (2019). Chronic methylphenidate treatment during adolescence has long-term effects on monoaminergic function. J. Psychopharmacol. 33, 109-121. doi: 10.1177/0269881118805494 
El Iskandrani, K. S., Oosterhof, C. A., El Mansari, M., and Blier, P. (2015). Impact of subanesthetic doses of ketamine on AMPA-mediated responses in rats: an in vivo electrophysiological study on monoaminergic and glutamatergic neurons. J. Psychopharmacol. 29, 792-801. doi: 10.1177/0269881115573809

El Mansari, M., Guiard, B. P., Chernoloz, O., Ghanbari, R., Katz, N., and Blier, P. (2010). Relevance of norepinephrine-dopamine interactions in the treatment of major depressive disorder. CNS Neurosci. Ther. 16, e1-e17. doi: 10.1111/j. 1755-5949.2010.00146.x

Federici, M., Geracitano, R., Bernardi, G., and Mercuri, N. B. (2005). Actions of methylphenidate on dopaminergic neurons of the ventral midbrain. Biol. Psychiatry 57, 361-365. doi: 10.1016/j.biopsych.2004.11.030

Gallegos, R. A., Lee, R. S., Criado, J. R., Henriksen, S. J., and Steffensen, S. C. (1999). Adaptive responses of $\gamma$-aminobutyric acid neurons in the ventral tegmental area to chronic ethanol. J. Pharmacol. Exp. Ther. 291, 1045-1053.

Golub, G. H., and Van Loan, C. F. (1996). Matrix Computation. 3rd Edn. Baltimore, MD: Johns Hopkins University Press.

Grace, A. A., and Bunney, B. S. (1983). Intracellular and extracellular electrophysiology of nigral dopaminergic neurons-1. Identification and characterization. Neuroscience 10, 301-315. doi: 10.1016/0306-4522(83) 90135-5

Grace, A. A., and Bunney, B. S. (1984). the control of firing pattern in nigral dopamine neurons: burst firing. J. Neurosci. 4, 2877-2890. doi: 10.1523/JNEUROSCI.04-11-02877.1984

Guiard, B. P., El Mansari, M., and Blier, P. (2008). Cross-talk between dopaminergic and noradrenergic systems in the rat ventral tegmental area, locus ceruleus and dorsal hippocampus. Mol. Pharmacol. 74, 1463-1475. doi: 10.1124/mol.108.048033

Hnasko, T. S., Hjelmstad, G. O., Fields, H. L., and Edwards, R. H. (2012). Ventral tegmental area glutamate neurons: electrophysiological properties and projections. J. Neurosci. 32, 15076-15085. doi: 10.1523/JNEUROSCI.3128 $-12.2012$

Hughes, E. W., Martin-Body, R. L., Sarelius, I. H., and Sinclair, J. D. (1982). Effects of urethane-chloralose anaesthesia on respiration in the rat. Clin. Exp. Pharmacol. Physiol. 9, 119-127. doi: 10.1111/j.1440-1681.1982.tb00788.x

Hyland, B. I., Reynolds, J. N. J., Hay, J., Perk, C. G., and Miller, R. (2002). Firing modes of midbrain dopamine cells in the freely moving rat. Neuroscience 114, 475-492. doi: 10.1016/s0306-4522(02)00267-1

Johnson, S. W., and North, R. A. (1992). Two types of neurone in the rat ventral tegmental area and their synaptic inputs. J. Physiol. 450, 455-468. doi: 10.1113/jphysiol.1992.sp019136

Jolliffe, I. T., and Cadima, J. (2016). Principal component analysis: a review and recent developments. Philos. Transact. A Math. Phys. Eng. Sci. 374:20150202. doi: 10.1098/rsta.2015.0202

Kassambara, A., and Mundt, F. (2017). Factoextra: Extract and Visualize the Results of Multivariate Data Analyses: An R Package in CRAN.

Kiyatkin, E. A., and Rebec, G. V. (1998). Heterogeneity of ventral tegmental area neurons: single-unit recording and iontophoresis in awake, unrestrained rats. Neuroscience 85, 1285-1309. doi: 10.1016/s0306-4522(98)00054-2

Lammel, S., Hetzel, A., Häckel, O., Jones, I., Liss, B., and Roeper, J. (2008). Unique properties of mesoprefrontal neurons within a dual mesocorticolimbic dopamine system. Neuron 57, 760-773. doi: 10.1016/j.neuron.2008.01.022

Li, W., Doyon, W. M., and Dani, J. A. (2012). Quantitative unit classification of ventral tegmental area neurons in vivo. J. Neurophysiol. 107, 2808-2820. doi: 10.1152/jn.00575.2011

Limpert, E., Stahel, W. A., and Abbt, M. (2001). Log-normal distributions across the sciences: keys and clues. BioScience 51, 341-352. doi: 10.1641/00063568(2001)051[0341:Indats]2.0.co;2

Liu, C.-L., Gao, M., Jin, G.-Z., and Zhen, X. (2012). GABA neurons in the ventral tegmental area responding to peripheral sensory input. PLoS One 7:e51507. doi: 10.1371/journal.pone.0051507

López-Jury, L., Meza, R. C., Brown, M. T. C., Henny, P., and Canavier, C. C. (2018). Morphological and biophysical determinants of the intracellular and extracellular waveforms in nigral dopaminergic neurons: a computational study. J. Neurosci. 38, 8295-8310. doi: 10.1523/JNEUROSCI.0651 $-18.2018$

Luo, A. H., Georges, F. E., and Aston-Jones, G. S. (2008). Novel neurons in ventral tegmental area fire selectively during the active phase of the diurnal cycle. Eur. J. Neurosci. 27, 408-422. doi: 10.1111/j.1460-9568.2007.05985.x
Mao, M., Nair, A., and Augustine, G. J. (2019). A novel type of neuron within the dorsal striatum. Front. Neural Circuits 13:32. doi: 10.3389/fncir.2019.00032

Margolis, E. B., Hjelmstad, G. O., Bonci, A., and Fields, H. L. (2005). Both kappa and mu opioid agonists inhibit glutamatergic input to ventral tegmental area neurons. J. Neurophysiol. 93, 3086-3093. doi: 10.1152/jn.00855.2004

Margolis, E. B., Lock, H., Hjelmstad, G. O., and Fields, H. L. (2006). The ventral tegmental area revisited: is there an electrophysiological marker for dopaminergic neurons? J. Physiol. 577, 907-924. doi: 10.1113/jphysiol.2006. 117069

Margolis, E. B., Mitchell, J. M., Ishikawa, J., Hjelmstad, G. O., and Fields, H. L. (2008). Midbrain dopamine neurons: projection target determines action potential duration and dopamine $\mathrm{D}(2)$ receptor inhibition. J. Neurosci. 28, 8908-8913. doi: 10.1523/JNEUROSCI.1526-08.2008

Marinelli, M., and McCutcheon, J. E. (2014). Heterogeneity of dopamine neuron activity across traits and states. Neuroscience 282, 176-197. doi: 10.1016/j. neuroscience.2014.07.034

Marinelli, M., and White, F. J. (2000). Enhanced vulnerability to cocaine self-administration is associated with elevated impulse activity of midbrain dopamine neurons. J. Neurosci. 20, 8876-8885. doi: 10.1523/JNEUROSCI.2023-08876.2000

Mejias-Aponte, C. A., Ye, C., Bonci, A., Kiyatkin, E. A., and Morales, M. (2015). A subpopulation of neurochemically-identified ventral tegmental area dopamine neurons is excited by intravenous cocaine. J. Neurosci. 35, 1965-1978. doi: 10.1523/JNEUROSCI.3422-13.2015

Mizuseki, K., and Buzsáki, G. (2013). Preconfigured, skewed distribution of firing rates in the hippocampus and entorhinal cortex. Cell Rep. 4, 1010-1021. doi: 10.1016/j.celrep.2013.07.039

Moghaddam, B., Adams, B., Verma, A., and Daly, D. (1997). Activation of glutamatergic neurotransmission by ketamine: a novel step in the pathway from NMDA receptor blockade to dopaminergic and cognitive disruptions associated with the prefrontal cortex. J. Neurosci. 17, 2921-2927. doi: 10.1523/JNEUROSCI.17-08-02921.1997

Morales, M., and Margolis, E. B. (2017). Ventral tegmental area: cellular heterogeneity, connectivity and behaviour. Nat. Rev. Neurosci. 18, 73-85. doi: 10.1038/nrn.2016.165

Morales, M., and Root, D. H. (2014). Glutamate neurons within the midbrain dopamine regions. Neuroscience 282, 60-68. doi: 10.1016/j.neuroscience.2014 05.032

Nair-Roberts, R. G., Chatelain-Badie, S. D., Benson, E., White-Cooper, H., Bolam, J. P., and Ungless, M. A. (2008). Stereological estimates of dopaminergic, GABAergic and glutamatergic neurons in the ventral tegmental area, substantia nigra and retrorubral field in the rat. Neuroscience 152, 1024-1031. doi: 10.1016/j.neuroscience.2008.01.046

Oosterhof, C. A., El Mansari, M., and Blier, P. (2014). Acute effects of brexpiprazole on serotonin, dopamine and norepinephrine systems: an in vivo electrophysiologic characterization. J. Pharmacol. Exp. Ther. 351, 585-595. doi: 10.1124 /jpet.114.218578

Oosterhof, C. A., El Mansari, M., and Blier, P. (2015). Asenapine alters the activity of monoaminergic systems following its subacute and long-term administration: an in vivo electrophysiological characterization. Eur. Neuropsychopharmacol. 25, 531-543. doi: 10.1016/j.euroneuro.2015. 01.006

Panin, F., Cathala, A., Piazza, P. V., and Spampinato, U. (2012). Coupled intracerebral microdialysis and electrophysiology for the assessment of dopamine neuron function in vivo. J. Pharmacol. Toxicol. Methods 65, 83-92. doi: $10.1016 /$ j.vascn.2012.01.003

Paxinos, G., and Watson, C. (2007). The Rat Brain in Stereotaxic Coordinates. 6th Edn. San Diego CA: Academic Press.

Petersen, P. C., and Berg, R. W. (2016). Lognormal firing rate distribution reveals prominent fluctuation-driven regime in spinal motor networks. eLife 5:e18805. doi: 10.7554/eLife.18805

Pinault, D. (1996). A novel single-cell staining procedure performed in vivo under electrophysiological control: morpho-functional features of juxtacellularly labeled thalamic cells and other central neurons with biocytin or Neurobiotin. J. Neurosci. Methods 65, 113-136. doi: 10.1016/0165-0270(95) 00144-1

Poulin, J.-F., Caronia, G., Hofer, C., Cui, Q., Helm, B., Ramakrishnan, C., et al. (2018). Mapping projections of molecularly defined dopamine neuron 
subtypes using intersectional genetic approaches. Nat. Neurosci. 21, 1260-1271. doi: 10.1038/s41593-018-0203-4

R Core Team. (2013). R: A Language and Environment for Statistical Computing. Vienna, Austria: R Foundation for Statistical Computing. Available online at: http://www.R-project.org/. Accessed July 1, 2020.

Root, D. H., Estrin, D. J., and Morales, M. (2018). Aversion or salience signaling by ventral tegmental area glutamate neurons. iScience 2, 51-62. doi: 10.1016/j. isci.2018.03.008

Rousseeuw, P. J. (1987). Silhouettes: a graphical aid to the interpretation and validation of cluster analysis. J. Comput. Appl. Math. 20, 53-65. doi: 10.1016/0377-0427(87)90125-7

Sanghera, M. K., Trulson, M. E., and German, D. C. (1984). Electrophysiological properties of mouse dopamine neurons: in vivo and in vitro studies. Neuroscience 12, 793-801. doi: 10.1016/0306-4522(84)90171-4

Shen, R.-Y. (2003). Ethanol withdrawal reduces the number of spontaneously active ventral tegmental area dopamine neurons in conscious animals. J. Pharmacol. Exp. Ther. 307, 566-572. doi: 10.1124/jpet.103.053371

Steffensen, S. C., Svingos, A. L., Pickel, V. M., and Henriksen, S. J. (1998). Electrophysiological characterization of GABAergic neurons in the ventral tegmental area. J. Neurosci. 18, 8003-8015. doi: 10.1523/JNEUROSCI.18-1908003.1998

Steffensen, S. C., Taylor, S. R., Horton, M. L., Barber, E. N., Lyle, L. T., Stobbs, S. H., et al. (2008). Cocaine disinhibits dopamine neurons in the ventral tegmental area via use-dependent blockade of GABA neuron voltage-sensitive sodium channels. Eur. J. Neurosci. 28, 2028-2040. doi: 10.1111/j.1460-9568.2008. 06479.x

Steffensen, S. C., Walton, C. H., Hansen, D. M., Yorgason, J. T., Gallegos, R. A., and Criado, J. R. (2009). Contingent and non-contingent effects of low-dose ethanol on GABA neuron activity in the ventral tegmental area. Pharmacol. Biochem. Behav. 92, 68-75. doi: 10.1016/j.pbb.2008.10.012

Stobbs, S. H., Ohran, A. J., Lassen, M. B., Allison, D. W., Brown, J. E., and Steffensen, S. C. (2004). Ethanol suppression of ventral tegmental area GABA neuron electrical transmission involves N-methyl-D-aspartate receptors. J. Pharmacol. Exp. Ther. 311, 282-289. doi: 10.1124/jpet.104. 071860

Taylor, A. M. W., Becker, S., Schweinhardt, P., and Cahill, C. (2016). Mesolimbic dopamine signaling in acute and chronic pain: implications for motivation, analgesia, and addiction. Pain 157, 1194-1198. doi: 10.1097/j.pain. 0000000000000494

Trulson, M. E., and Trulson, T. J. (1987). Recording of mouse ventral tegmental area dopamine-containing neurons. Exp. Neurol. 96, 68-81. doi: 10.1016/00144886(87)90169-5
Ungless, M. A., and Grace, A. A. (2012). Are you or aren't you? Challenges associated with physiologically identifying dopamine neurons. Trends Neurosci. 35, 422-430. doi: 10.1016/j.tins.2012.02.003

Ungless, M. A., Magill, P. J., and Bolam, J. P. (2004). Uniform inhibition of dopamine neurons in the ventral tegmental area by aversive stimuli. Science 303, 2040-2042. doi: 10.1126/science.1093360

Valenti, O., and Grace, A. A. (2010). Antipsychotic drug-induced increases in ventral tegmental area dopamine neuron population activity via activation of the nucleus accumbens-ventral pallidum pathway. Int. J. Neuropsychopharmacol. 13, 845-860. doi: 10.1017/s1461145709990599

Valenti, O., Gill, K. M., and Grace, A. A. (2012). Different stressors produce excitation or inhibition of mesolimbic dopamine neuron activity: response alteration by stress pre-exposure. Eur. J. Neurosci. 35, 1312-1321. doi: 10.1111/j.1460-9568.2012.08038.x

Vu, Q. V. (2011). ggbiplot: A ggplot2 Based Biplot. R Package 0.55. Available online at: http://github.com/vqv/ggbiplot. Accessed July 1, 2020.

Ward, J. H. (1963). Hierarchical grouping to optimize an objective function. J. Am. Stat. Assoc. 58, 236-244. doi: 10.1080/01621459.1963.10500845

Yamaguchi, T., Qi, J., Wang, H.-L., Zhang, S., and Morales, M. (2015). Glutamatergic and dopaminergic neurons in the mouse ventral tegmental area. Eur. J. Neurosci. 41, 760-772. doi: 10.1111/ejn.12818

Yamaguchi, T., Wang, H.-L., Li, X., Ng, T. H., and Morales, M. (2011). Mesocorticolimbic glutamatergic pathway. J. Neurosci. 31, 8476-8490. doi: 10.1523/JNEUROSCI.1598-11.2011

Yim, C. Y., and Mogenson, G. J. (1980). Electrophysiological studies of neurons in the ventral tegmental area of Tsai. Brain Res. 181, 301-313. doi: 10.1016/00068993(80)90614-9

Zhang, D., Yang, S., Jin, G.-Z., Bunney, B. S., and Shi, W.-X. (2008). Oscillatory firing of dopamine neurons: differences between cells in the substantia nigra and ventral tegmental area. Synapse 62, 169-175. doi: 10.1002/syn.20479

Conflict of Interest: The authors declare that the research was conducted in the absence of any commercial or financial relationships that could be construed as a potential conflict of interest.

Copyright (C) 2020 Di Miceli, Husson, Ruel, Layé, Cota, Fioramonti, Bosch-Bouju and Gronier. This is an open-access article distributed under the terms of the Creative Commons Attribution License (CC BY). The use, distribution or reproduction in other forums is permitted, provided the original author(s) and the copyright owner(s) are credited and that the original publication in this journal is cited, in accordance with accepted academic practice. No use, distribution or reproduction is permitted which does not comply with these terms. 